\title{
S-VLWIC: Secure Visible Light based Wireless Communication using Location Information
}

\author{
Suparna Biswas \\ Department of Computer Science \& Engineering, \\ Maulana Abul Kalam Azad University of Technology, WB.
}

\begin{abstract}
With the rapid advancement in semiconductor technology and wireless links, indoor lighting sources are prevalently getting dominance of white light emitting diodes (LEDs) overincandescent and fluorescent bulbs over the years. This trend leads to energy efficiency along with less carbon emission with a support of multi functionalities such as datatransmission along with illumination. As multiple LEDs are there at ceiling for uniform lighting, those LEDs can be used to get location information of user with smartphone having photosensor for more accurate indoor positioning in comparison with standard GPS system. User location based data transmission ensures authentication and access control over data. Data getting streamed through visible light remain confidential within a physical confinement as light cannot penetrate through walls. Moreover, once authentication based on location information and device ID is done, data streaming through visible light commences and no adversary can modify data. This ensures data integrity as well. Hence sensor based applications especially healthcare system that needs interference free real-time data streaming maintaining privacy of patients' health data, may demand immensely for such low overhead secure wireless communication using visible light.
\end{abstract}

\section{General Terms}

Security, location, RSSI, visible light, wireless communication etc.

\section{Keywords}

Secret key exchange, Visible light communication, security, low overhead, confidentiality, integrity etc.

\section{INTRODUCTION}

With the advent of LEDs as a result of fast advancement of semiconductor technology, communication and illumination comes in a package providing wireless communication through optical channel specifically high speed data download without the hazards of multipath fading, electromagnetic interference, licencing of bandwidth, eavesdropping etc. Other driving factors that enhanced the fast development of Visible Light Communication (VLC) are availability of small, lightweight, cheap LEDs with modulation capability giving dimming advantage to encapsulate data while illuminating in power aware way, compared to traditional fluorescent and incandescent lights which have lower lifetime and no dimming advantage [1]. The general architecture of Visible Light Communication System is very simple as components are LED transmitters (Tx), receivers with photodetector (Rx) and optical channel through which transmitter encodes and sends modulated data with light and receiver decodes the received signal and converts from optical to electrical domain.

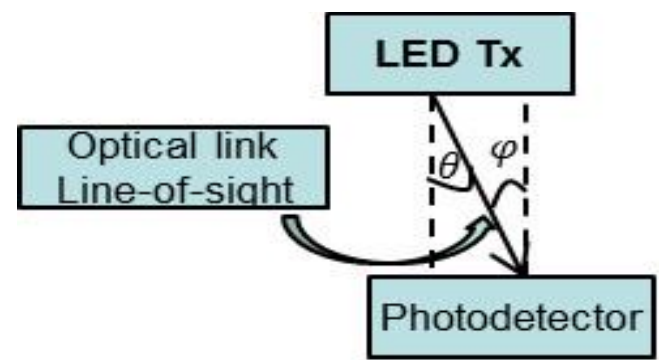

Figure 1. Optical communication involving LED Tx, Rx with photodetector and optical link

In figure 1, LED Tx transmits data to receiver using optical link in between and communication is line-of-sight with equal transmitter $(\theta)$ and receiver angle $(\varphi)$. But it is crucial to implement illumination and communication both with equal weightage because there is a trade-off between these two efficient illumination depends on wide divergence angle $(\theta)$ as more the divergence angle, longer the radius of covered area and efficient communication depends on narrow divergence angle $(\theta)$ as less the divergence angle higher the range of data transmission. There are very few works that optimizes these two leaving a very promising research scope to explore in future.

Visible Light Communication - the name reflects light is visible but it wraps data within itself and makes it invisible to the crowd with normal eyes. So, encapsulation is inherent in characteristics of visible light from LED. Light cannot penetrate walls, so physical security is ensured within a confinement - confidentiality unless one is coming with a photodetector in direct line of sight (LoS). Once data transmission starts, no one can change or modify data being transmitted through optical channel which is out-of-band [ 2 ] in nature. Thus integrity can be implemented. And receiver position and receiver ID based data transmission or services ensures authentication of legitimate user. So, full security solution comes in a package without additional encryption, decryption or any other computation complexities. Moreover, to encrypt and decrypt data before transmission and receive respectively need time which increases delay in response. Healthcare is a real time system where instant response and prompt action from care givers e.g. doctors / nurses can save lives of patients in critical condition. So, Secure Visible Light based Wireless Communication (S-VLWiC) can be characterized with followings - 


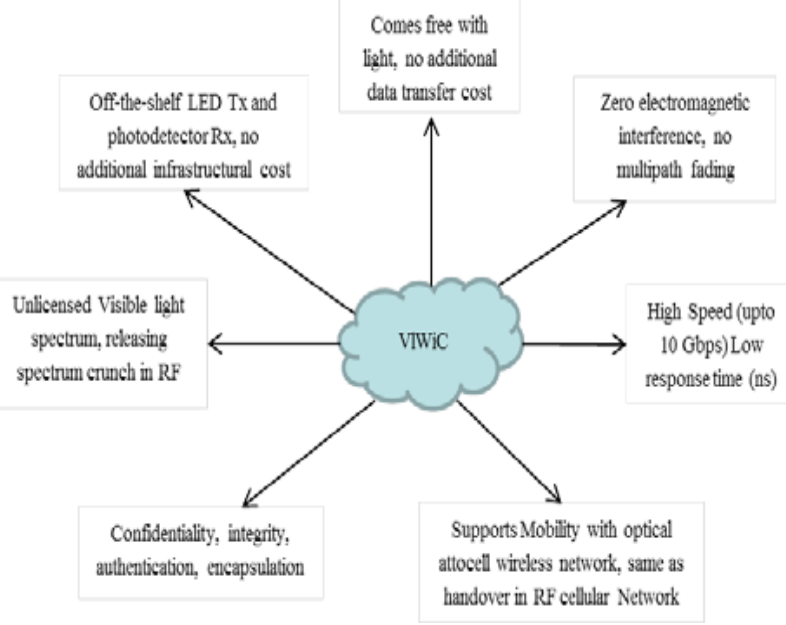

Figure 2. Visible Light based Wireless Communication with unique features and advantages

In VLWiC, transmitters are at fixed locations generally at ceiling in indoors and receiver can be at any position at a certain height above floor. To get position information of receiver carried by user is a popular and much investigated problem and at outdoors using Global Positioning System (GPS), location information can be known but with an approximation of few meters. GPS cannot be applied to indoors due to several limitations as given below:

- Not suitable for indoors-a device must receive signals from a no. of GPS satellite-often not possible in indoors

- Not still available a cheap, accurate and widely available positioning system using radio system based on wireless Local Area Networks (LANs)

- Lacks a reliable and simple positioning system as receiver receives signal after multiple reflections -

hence difficult to estimate distance or direction of the transmitter analysing received signal

- Accuracy of positioning is about meters- in indoors room sizes are small (e.g. $5 \mathrm{~m}^{2}$ ) hence this error cannot be acceptable. In indoors, position accuracy is most important for different applications e.g. tracking a person or an object, giving navigation support or position based data transmission, any services in indoors require high position accuracy for proper functioning.

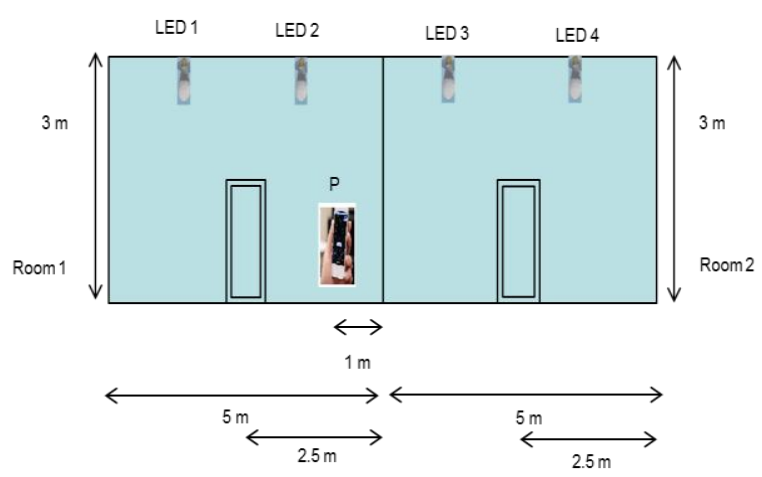

Figure 3: Front view of 2 adjacent same size room equipped with LED Txs at fixed location and Rx at any position inside room

In this figure, the position of a person with receiver is shown in room 1 . He wants to download some specific data which may need privacy e.g. patient's health records. The receiver and transmitters will be synchronized, then receiving $\mathrm{Tx}$ codes, Tx coordinates, applying RSSI and trilateration technique, receiver calculates its position and sends to server for verification through Txs. If the position accuracy is less than a meter, the person will be identified to be in room 1 , but of position accuracy is more than a meter, his position may be inaccurate by either side (left or right), in case of right side, his position will be wrongly identified as in room 2 and server will send data for transmission through LED 3 and LED 4. If any eavesdropper is in room 2 he can easily have access to the data violating confidentiality requirement of data and the desired person not receiving it. Position accuracy in indoor positioning system must be very precise and using Visible Light Communication, there are many systems reported with high accuracy in few $\mathrm{cm}$ or $\mathrm{mm}$ [4]. To calculate position, real optical channel needs to be simulated properly as real environment is dynamic and volatile, varying ambient light noise level, reflections in walls or any objects inside room, receiver mobility etc. affects RSSI based positioning in terms of accuracy.

Rest of the paper is organized as follows: section 2 describes related works, section 3 reports about different positioning techniques, section 4 details out different applications, section 5 explains secure data transmission framework along with algorithm and flowchart using Visible light, finally whole paper is concluded in section 6 .

\section{RELATED WORKS}

LED based wireless communication has attracted many researchers to explore its advantages for high speed down streaming of data for different applications [4]. But success of this comes to a question at night time or to the scenario when light is not always ' $\mathrm{ON}$ ' e.g. while watching movies or multimedia application in smartphones or Ipad or tablets, people often prefers for the room to be dark or while giving power point presentation if a video file has to be downloaded or play using VLC channel, LED must be 'ON' which not real scenario as room is usually dark. VLC is preferred to be a green technology as power consumption of LEDs are less but communication comes free when LEDs are ' $O N$ ' for illumination purpose but if lights need to be kept 'ON' only for communication purposes then it adds cost (night time). In [5], authors address the issue which is very much related to VLC research for communication and that is, how communication can be successfully done in absence of light i.e. if lights are 'OFF'? Minimum light intensity required to communicate has been defined and this state has been designated as "lights-off" state. The light intensity at this level is zero from human perception but maintained at very low level and this power level from VLC transmitters may vary based on ambient shot noise. For varying data rates $(20 \mathrm{~kb} / \mathrm{s}$, $100 \mathrm{~kb} / \mathrm{s}, 2 \mathrm{Mb} / \mathrm{s}$ ), required light intensity (lx) varies between 10-5 to 101 for a wide range of ambient illumination (lx) from 10-6 to 106 . Main contribution is to prove the feasibility of data communication with several $\mathrm{Mb} / \mathrm{s}$ data rate using low complexity devices focusing on data coverage. VLC based communication is 'green' for another important property of LED - dimming control. Though there are many other advantages of dimming LEDs e.g. fulfilling ambience illumination demand, less number of LEDs to be maintained, increased lifetime of illuminating sources as less heat will be generated, improvement on light source efficacy (ratio of luminous flux to power), main purpose is to save additional energy consumption and to reduce cooling load on HVAC (heating, ventilation and air conditioning) [ 6 ]. Though communication gets hampered due to dimming as data rate is 
inversely proportional to duty cycle of PWM signal and if duty cycle is low, high data rate often becomes difficult to implement in actual system [ 7 ]. There are already different adaptive techniques in place to compensate dimming effect on communication [ 6 ]. U.S. Department of energy [8] describes and specifies about LED dimming characteristics, challenges to implement in real world as there are wide source of LEDs 2 and dimmer characteristics so general assumptions cannot be made for all types etc. LED characteristics make it suitable for dimming and these are its eminent property to be ' $\mathrm{ON}$ ' for varying current, non-flickering, no noise generation, longer lifetime due to less current and low temperature. In [9], an Indoor Positioning System (IPS) using Visible Lights applies Dual -Tone Multi Frequency (DTMF) technique as the system is able to distinctly identify signals transmitted by adjacent LEDs in both time and frequency domains avoiding interchannel interference and clock synchronization. To get estimated receiver position, both LED transmitter IDs and location coordinates are used and time frequency analysis of received RSSI is done to get that. Based on received LED ID from which highest RSSI is received signifying that LED Tx is nearest to receiver, by mapping ID in database, estimated position of receiver can be obtained. To accurately calculate position, channel gain of individual channel is obtained. Real scenario has been simulated using Monte Carlo based simulation and position error has been calculated for different receiver positions within experimental space to find position error is minimum in the middle and increases as the receiver moves away in any direction. In [10], an Indoor Positioning System (IPS) using Visible Lights applies Dual one Multi Frequency (DTMF) technique as the system is able to distinctly identify signals transmitted by adjacent LEDs in both time and frequency domains avoiding inter-channel interference and clock synchronization. To get estimated receiver position, both LED transmitter Ids and location coordinates are used and time frequency analysis of received RSSI is done to get that. Based on received LED ID from which highest RSSI is received signifying that LED Tx is earest to receiver, by mapping ID in database, estimated position of receiver can be obtained. To accurately calculate osition, channel gain of individual channel is obtained. Real scenario has been simulated using Monte Carlo based imulation and position error has been calculated for different eceiver positions within experimental space to find position error is minimum in the middle and increases as the receiver oves away in any direction. In [3], different indoor positioning techniques, theories behind and comparative analysis among techniques has been given for wireless systems. Li-Fi is wireless system based on LEDs hence these techniques are equally been applied for Visible Light Positioning Systems. This paper categorizes different position information e.g. physical location, relative location, Symbolic location and absolute location. Also this paper highlights four different ways of positioning among which self-positioning is mostly done where measuring unit is receiver and mobile, and calculates own position based RSS from different transmitters at fixed locations. The receiver can be a smartphone or aipad with photosensor in real scenario. Indoor positioning system and position based different application has been designed combining VLC for downlink and RF/IR for uplink in indoors, some of which are already in place.

Another important and popular aspect of an open wireless system ( e.g. Wi-Fi) is that people can be able to connect to internet through access points or hot-spots using their device

e.g. smartphone and can continue to having access to it even he/she is moving e.g. walking through corridors or within the room. VLC based mobile wireless network are becoming a prospective future technology as reported in [1], [11], [12]. In [11], authors have addressed this issue and have defined a receiver sensitivity value which sets a minimum received power below which receiver will be disconnected from LED transmitters i.e. optical link between Rx and Tx will be lost. If receiver and transmitter are out of their field of view (FoV) then receiver is considered to be out of coverage and hence will be disconnected. So designing VLC based wireless networking system ensuring full coverage within a defined space optimizing number of LED transmitters and minimizing overlap regions covered by multiple LEDs is an ongoing attractive topic to researchers. Though light based wireless networking is a much explored area by researchers, pure VLC communication is still far away from reality because of uplink issue. Light is always in downward direction facilitating downlink hence for uplink, Wi-Fi, IR are used. In [12], authors claimed to achieve bi-directional high speed optical wireless line-of-sight communication using visible LED for downlink and IR LED for uplink. They achieved $250 \mathrm{Mbit} / \mathrm{s}$ for both downlink and uplink considering ambient light noise within a $12 \mathrm{~m}^{2}$ room area and non-line-of-sight communication.

\subsection{Positioning Techniques}

Positioning or localization of resources (people or object) has a long history and there are different techniques to estimate locations e.g. Triangulation, Scene analysis and proximity [15]. Among these, triangulation is based on location calculation using properties of triangles as the name reflects and can be of two types as per the difference in methods: lateration-distance measurement, angulation-angle measurement. For distance measurement there must be some reference points, number of which may vary based on the dimension of position estimation. e.g. 3 non-collinear fixed reference point location coordinates must be known for 2-D position information and 4 non-collinear fixed point location coordinates must be known for 3-D position information. For measuring distance from reference point which are LED transmitters in Visible Light Positioning, there are different methods, some of which are - i) RSSI : Received Signal Strength Intensity technique in which distance of a receiver from reference points is calculated by measuring received signal strength at receiver from reference point because signal strength decreases with increase in distance of receiver from reference point and vice versa. If distance between receiver and reference point is $\mathrm{r}$, signal is attenuated by a factor of $\frac{1}{r^{2}}$. ii) TOA (Time of Arrival): Time of arrival of signal from a reference point is measured i.e. one way signal propagation and accordingly distance can be measured if speed of signal is known. To apply trilateration, atleast 3 such distances has to be measured from 3 different reference points iii TDOA (Time Difference of Arrival): There are two signal transmitters time synchronized and send signals to a receiver which measures the time difference between arrival of two different signals and accordingly measures the distance. Iv) AOA (Angle of Arrival): To estimate position of an object, intersection of the number of angle direction lines is found and each such line is obtained by the circular radius from a base station, here LED transmitter. A comparative study among all these techniques is given below summarizing, advantages and drawbacks. Following is the pictorial illustration of RSSI technique. 


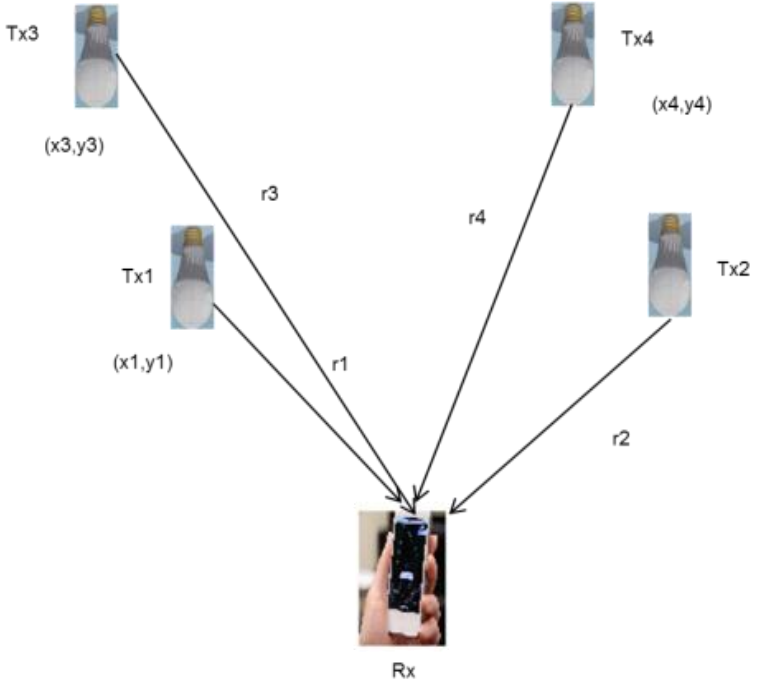

Figure 7: 4 Txs at ceiling, $R x$ at a position $(x, y, z)$ where $\mathrm{z}=\mathrm{h}$, at a fixed height above floor, receives signal from all 4 Txs

At receiver, signal from each Tx is separately measured and distance from each $\mathrm{Tx}(\mathrm{r})$ is calculated. Now Txs are at known coordinates $(\mathrm{x} 1, \mathrm{y} 1),(\mathrm{x} 2, \mathrm{y} 2),(\mathrm{x} 3, \mathrm{y} 3)$ and $(\mathrm{x} 4, \mathrm{y} 4)$ for $\mathrm{Tx} 1, \mathrm{Tx} 2$, $\mathrm{Tx} 3$ and Tx4 respectively. For trilateration, 3 reference points are needed. Here signal strength received from $\mathrm{Tx} 3$ is minimum and $\mathrm{r} 3$ is maximum, hence $\mathrm{T} \times 3$ is discarded. Applying trilateration using following formula, 2-D position $(\mathrm{x}, \mathrm{y})$ of $\mathrm{Rx}$ can be estimated as height is fixed and known, so putting $\mathrm{z}=\mathrm{h}$, estimated position $(\mathrm{x}, \mathrm{y}, \mathrm{z})$ can be obtained.

Table 1. Analytical Comparison of Different Positioning Techniques for Trilateration

\begin{tabular}{|c|c|c|}
\hline & To be known & Problem \\
\hline RSSI & $\begin{array}{l}\text { - Channel characteristics } \\
\text { - Optical Power from each LED }\end{array}$ & $\begin{array}{l}\text { - Transmitted optical power is } \\
\text { unpredictable, based on LED } \\
\text { and dimming } \\
\text { - If someone or something is } \\
\text { partially blocking path of light }\end{array}$ \\
\hline TOA & $\begin{array}{l}\text { - Requires transmitted signals to } \\
\text { be synchronized very accurately }\end{array}$ & $\begin{array}{l}\text { - Accurate transmitter } \\
\text { synchronization }\end{array}$ \\
\hline TDOA & $\begin{array}{l}\text { - At least } 2 \text { receivers with } \\
\text { accurately known distance } \\
\text { between } \\
\text { - TDOA of signals reaching } 2 \text { Rxs } \\
\text { gives info. about path length } \\
\text { from transmitter }\end{array}$ & $\begin{array}{l}\text { - Signal propagates at speed } \\
\text { of light } \\
\text { - Distance between Tx and } \\
\text { Rx is small } \\
\text { - Extremely accurate time } \\
\text { measurement is required }\end{array}$ \\
\hline $\mathrm{AOA}$ & $\begin{array}{l}\text { - LoS between Tx and Rx } \\
\text { - Diffuse lights are very small } \\
\text { hence error negligible }\end{array}$ & - blocking \\
\hline
\end{tabular}

\section{APPLICATIONS}

Users with smartphones or Ipad generally spend $80-90 \%$ of time during day-time in indoors which are having smart lighting nowadays with LEDs. LEDs have dimming property and using this, data stream can be transmitted as light rays as this flicker is not recognizable with naked human eyes. Smartphones having photosensors can receive data if it is within coverage of LED transmitters. Advantages are many fold - High Speed Data download comes free with light (when normally lights are $\mathrm{ON}$ ), Visible light spectrum used for download does not crowd already scarce RF licensed spectrum, No electromagnetic interference, No multipath fading due to penetration through walls, No additional infrastructural cost and inherent security. Thus, researchers are attracted to design a range of systems/applications, based on Visible Light Communication, some of which are already in place.

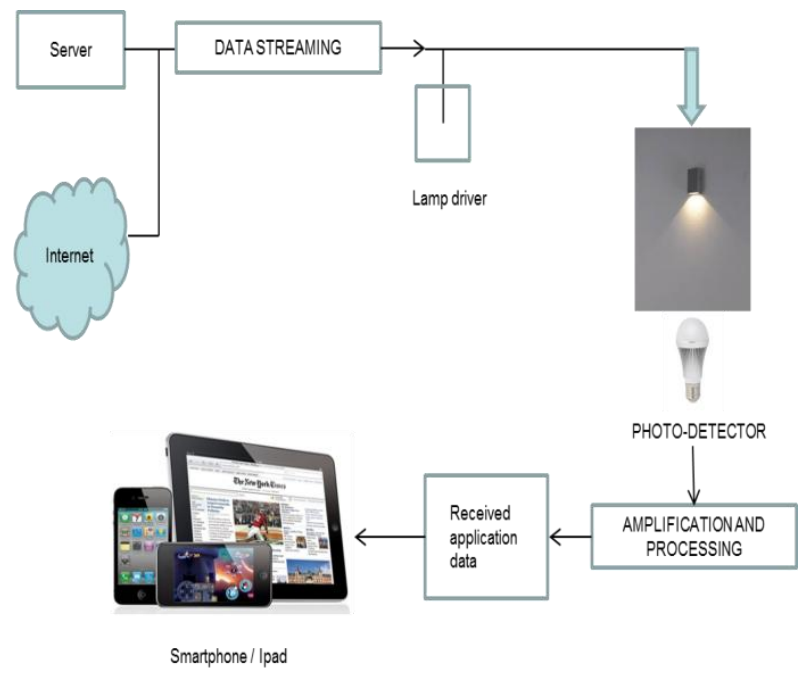

Figure 8. Working Model of VLC based Application/Service System

In figure 8, general model of LED transmitter and photodetector receiver based downstreaming / download and display of data is shown e.g. watching movies, listening to music. Location coordinate of LED transmitter is known, it can work as a reference point to find location of receiver. Receiver can move which will be identified and accordingly corresponding LED transmitter will transmit data and hence, seamless connectivity with mobility can be provided. While receiver moves, if its position is identified with high accuracy, then navigation can be done within the environment. Like GPS, the communication here is from transmitter to receiver. GPS in indoors is not feasible as receiver has to receive sufficiently strong signal from at least three Base stations and this is not possible due to multipath fading. For indoor navigation, high precision accuracy is required as e.g., say in a art gallery, one person is in front of picture of 'Monalisa', but due to position inaccuracy his current position is 2-3 meters away in front of a picture of 'Titanic', the ship and in his audio jack its description starts. Or in a hospital, rooms are identical and adjacent, hence position inaccuracy of few meters may give incorrect navigation information.

VLP gives high position accuracy in natural ambience with noise i.e. indirect sunlight or any background lights and hence suitable for navigation, tracking, position based services [16][17]. 
Table 2. Applications of VLC with Justifications

\begin{tabular}{|c|c|c|}
\hline Systems/applications & Advantage & Function \\
\hline Healthcare & No EM interference & $\begin{array}{l}\text { High speed download using } \\
\text { optical channel }\end{array}$ \\
\hline Aviation & No cabling, reduce weght & LED based Entertainment \\
\hline $\begin{array}{l}\text { Underwater } \\
\text { Communications }\end{array}$ & Suitable for shat-range & Navigation, searching \\
\hline Transport \& tr afic & $\begin{array}{l}\text { Car head and tail lights, car- } \\
\text { to-ca communication }\end{array}$ & $\begin{array}{l}\text { street lights are LEDs, } \\
\text { roadside traffic to ca } \\
\text { communication }\end{array}$ \\
\hline $\begin{array}{l}\text { Location based services i.e. } \\
\text { shopping mals or Art gallery } \\
\text { or museum }\end{array}$ & $\begin{array}{l}\text { High position accuracy, } \\
\text { correct information }\end{array}$ & $\begin{array}{l}\text { Timely and location based } \\
\text { information receiving }\end{array}$ \\
\hline
\end{tabular}

\section{PROPOSED APPLICATION OF USER LOCATION BASED GREEN SECURE DATA TRANSMISSION USING VLC}

As in touched in section I, security of VLP systems with simplicity, low complexity and fast response time as no additional encryption-decryption calculations are added, hence real time systems e.g. banking system where data cannot be disclosed publicly are in demand of such positioning systems. For private key encryption-decryption, secret key needs to be shared between sender and receiver. Here, for secret key transmission between sender (admin) and receiver (employee) for financial transactions in banking, secure VLC channel is used after authenticating the receiver by having its current position information and unique receiver ID based on which access control can be implemented. Banking system is an indoor environment with fixed light fixtures for illumination which can be combined with data transmission if lighting elements are replaced with LEDs, thus power efficiency can be achieved. Following is the block diagram of proposed position based secure data transmission system.

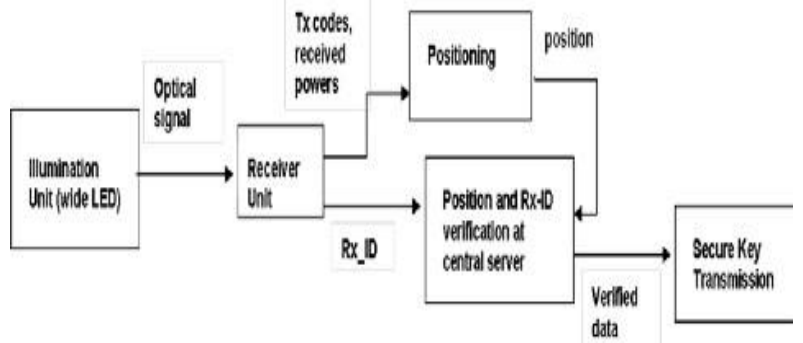

Figure 6. Block Diagram of Secret Key Transmission

In figure 6 , receiver receives light emitted form LED transmitters, sends $\mathrm{Tx}$ codes, received powers, to the positioning unit which may be within receiver (hence given

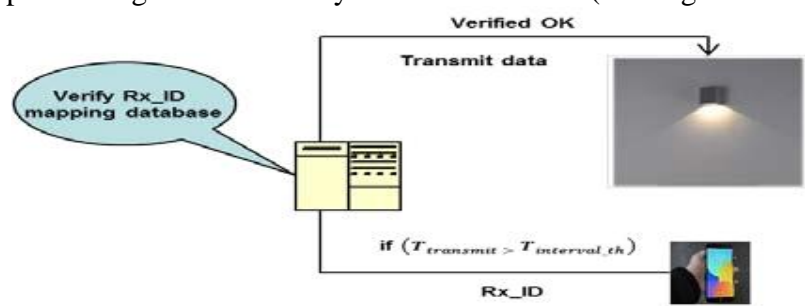

the term receiver unit) or may be separate unit. Estimated position (EP) from positioning unit and Rx_ID from receiver is sent to central server for verification based on saved database. If verified correctly then secret key will be transmitted through LEDs to the receiver.

Now the question is after data transmission has started based on authentication of receiver verifying its current location and Rx_ID, how can it be ensured that only the legitimate user is receiving the data, not anyone else e.g. an eavesdropper or an illegitimate user? The advantage of VLC is that light cannot penetrate through walls hence information transmitted through lights are confined and secure within a room but how to prevent information leakage or eavesdropping within a room? The advantage here again is that receiver (smartphone / Ipad) is carried by user himself hence any other person's entry within the room is observable but what if the legal user leaves the room after data streaming has started? To prevent such scenario to happen that may violate confidentiality of data, following prevention methods has been proposed as probable solution.

i) A threshold of data transmission time interval can be defined per system, per application basis. If data transmission time is more than this threshold, receiver will send its ID to server where it will be verified against saved ID which started receiving data. If current Rx_ID is same with that saved in database as starting $\mathrm{Rx}$ _ID then data transmission will continue otherwise will be stopped generating and broadcasting an 'alarm message'.

ii) ii)Another way of identifying someone's presence inside room is by checking the received power as it will change due to reflected power by the person's body if ambient light is remaining same. The change in received power may be effect of change in ambient light or any other reason and to distinguish it is a real challenge. As we have already mentioned, threshold of data transmission time interval can be varied per system per application basis, e.g. in healthcare patient record e.g. ECG record is high volume of data which may take significant amount of time in comparison to that to transmit a secure key which is small in size in banking application.

Figure 7. Secure data transmission: (a) legal user receives data (b) Secure VLC based data transmissionsystem identifies illegal user, data transmission is denied 


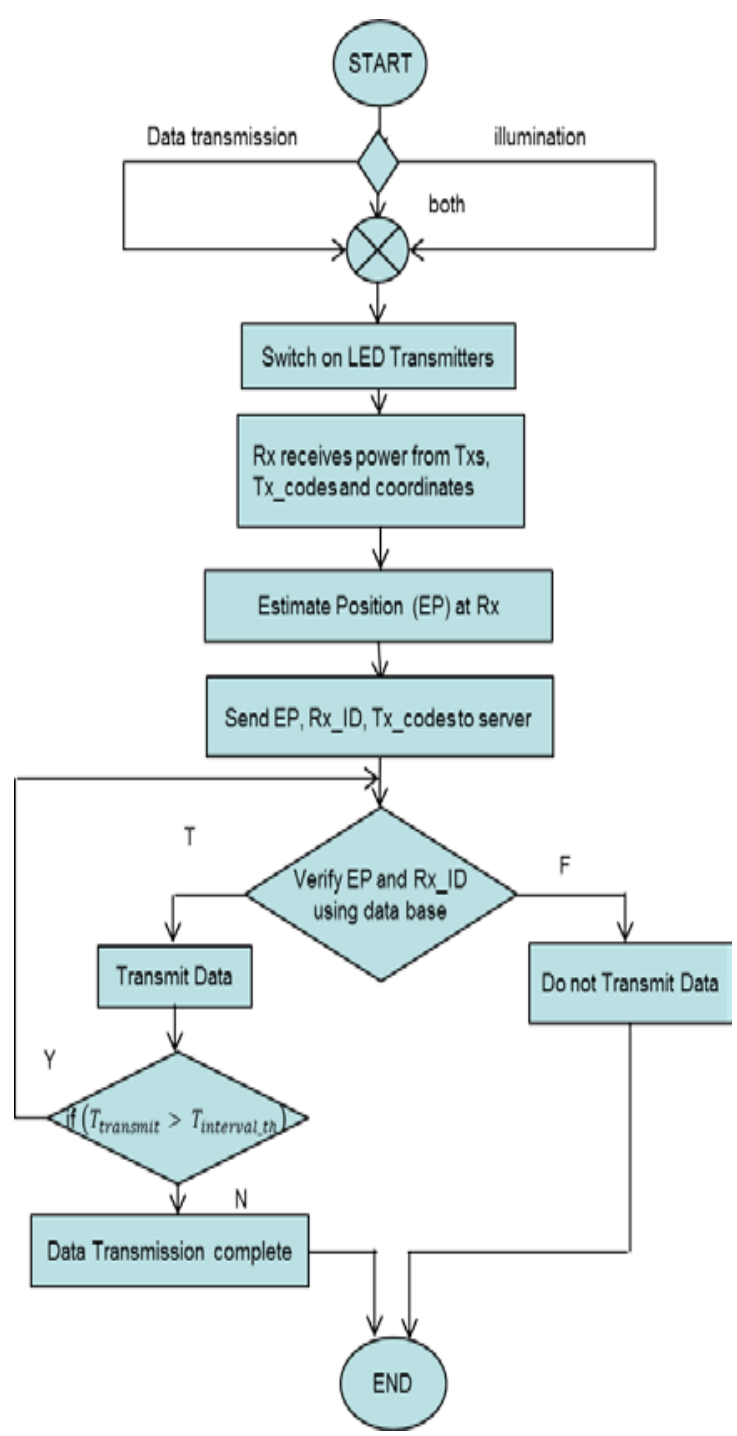

/* Algorithm for user Triggered Secure Key Transmission *I

1. User triggers key transmission

2. Estimate Rx_position (EP)

3. Send EP, Rx ID and Tx codes to server

4. Verify EP against Tx_codes using ceiling map

5. VerifyRx_ID in database

5.1 if ( verification $==$ TRUE)

5.1.1 start keytransmission

5.2 else

5.2.1 set alarm 'authentication violated'

5.2 .2 do not transmit key

5.2.3 Go to END

6.iff $\left(T_{\text {transmit }}>T_{\text {intv }}\right)$

6.1 Send Rx_ID to the server

6.2 Repeat step 5,6

7. else

7.1 if $($ Transmission $==$ complete $)$

8. END

Figure 8. Flowchart of Secure Key Transmission using VLP

Figure 8 shows the flowchart of proposed secure data transmission system. After authenticating the user based on current location information and receiver ID he is carrying to receive data transmission starts with a periodic verification of receiver ID unless data is fully transmitted. Algorithm described in figure 9 also illustrates this.

\section{CONCLUSION}

LED Based secure session key transmission will lead to low power consumption than using incandescent light as dimmed or 'OFF' LED still can transmit data. Unicast secure communication is feasible ensuring confidentiality and integrity once authentication of receiver is done. For authentication location location information of receiver is used. Data from server can be downloaded fast to the legitimate user by exploring inherent property of visible light.Applications that demand real time secure data download e.g. healthcare, financial or banking sector, military or defence etc. Can hugely explore this possible use of $\mathrm{Li}-\mathrm{Fi}$.

\section{REFERENCES}

[1] A. Sevincer, "LIGHTNETS: Smart Lighting and Mobile Optical Wireless Networks - A Survey", Communications Surveys and Tutorials, Vol. 15, issue 4.

\section{Figure9.Algorithm Description.}

[2] R. Roman, J. Lopez, "KeyLED - Transmitting sensitive Data Over Out-of-band Channel in Wireless Sensor Networks", in proceedings of 5 IEEE International Conference on Mobile Adhoc and Sensor Systems (MASS 2008), pp. 796-801, Atlanta, GA, 2008.

[3] L.Li, P.Hu, C.Peng, G.Shen, F.Hao, "Epsilon: a Visible Light based Positioning System", in proceedings of 11 th USENIX Conference on Networked Systems Design and Implementation, NSD'14, Pages 331-343, 2014.

[4] H. Liu, P. Banerjee, J. Liu, “, Survey of Wireless Indoor Positioning", IEEE transactions on Systems, man and cybernetics-part c: applications and reviews, vol. 37, no. 6, November 2007

[5] T. Borogovac, M.B. Rahaim, M. Tuganbayeva, and T.D.C. Little, “"Lights-off" Visible Light Communications", MCL Technical Report No. 0907-2011.

[6] F.Zafar, D. Karunatilaka,R. Parthiban, "Dimming Schemes for Visible Light Communication: the $6^{\text {th }}$ State of Research", IEEE Wireless Communications, Volume: 22, Issue: 2, April 2015. 
[7] Z. Wang, W. D.Zhong,C. Yu, J. Chen, C. P. Shin Francois, W. Chen, "Performance of dimming control scheme in visible light communication system", Vol. 20, No. 17 , OPTICS EXPRESS, 13 August 2012 .

[8] M.Poplawski, "Energy Efficiency and Renewable Energy, LED Dimming": What you need to know, DOE SSL program, U.S. Department of Energy.

[9] P. Luo, M. Zhang, X. Zhang, G. Cai, D. Han and Q. Li, An Indoor Visible Light Communication Positioning System Using Dual-Tone Multi Frequency Technique, $2^{\text {nd }}$ International Workshop on Optical Wireless Communications (IWOW), 2013.

[10] B. Schoettle, M.Sivak, Y. Fujiyama, "LEDs and Power Consumption of Exterior Automotive Lighting: Implicationsfor Gasolineand Electric Vehicles", The University of Michigan, Transportation Research
Institute, Ann Arbor, Michigan 48109-2150, U.S.A., Report No. UMTRI2008-48, October 2008.

[11] A.Burton, Z. Ghassemlooy, S.Rajbhandari,S.K.Liaw, Design and Analysis of an angular-segmented fullmobility visible light communications receiver", Transactions on Emerging Telecommunications Technologies.

[12] G.Cossu, R.Corsini, E.Ciaramella, "High-Speed Bidirectional Optical Wireless System in NonDirected Line-of-Sight Configuration", Journal of Lightwave Technology, Vol. 32, No. 10, May 15, 2014.

[13] K.Dividis, "Design and Prototyping of a Visible Light Indoor Positioning System", Philips Research Eindhoven, Technical note TN-2007-00115. 